\title{
Electric Field Driven Accumulation and Alignment of CdSe and CdTe Nanorods in Nanoscale Devices
}

\author{
Zonghai Hu, Michael D. Fischbein, Claudia Querner, and Marija Drndić*
}

Department of Physics and Astronomy, University of Pennsylvania, 209 South $33^{\text {rd }}$ St., Philadelphia, PA 19104
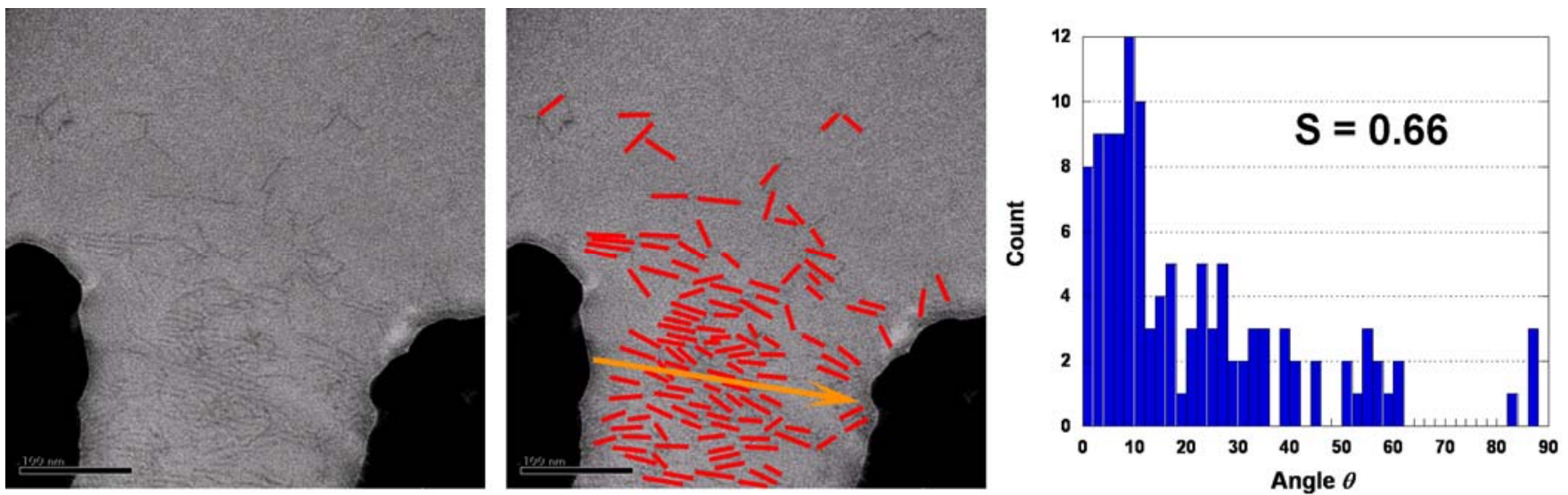

Figure S1. Example analysis of the alignment of CdSe NRs, shown in Figure 2d of the main article, (concentration $\sim 10^{13} \mathrm{rods} / \mathrm{ml}$ ) induced by a high electric field $\left(E=2.4 \times 10^{7} \mathrm{~V} / \mathrm{m}\right)$. Left: original TEM image, middle: determination of the angle of the rods (using ImageJ software) with respect to the applied electric field (yellow arrow), right: histogram of the angle distribution. From this histogram, we calculated a nematic order parameter value, $S$, of 0.66 . This value is actually an underestimation; the NRs outside of the gap have been included, even though the field is most likely not uniform in this region. Furthermore, within the gap, there are field inhomogeneities due to the electrode roughness. 


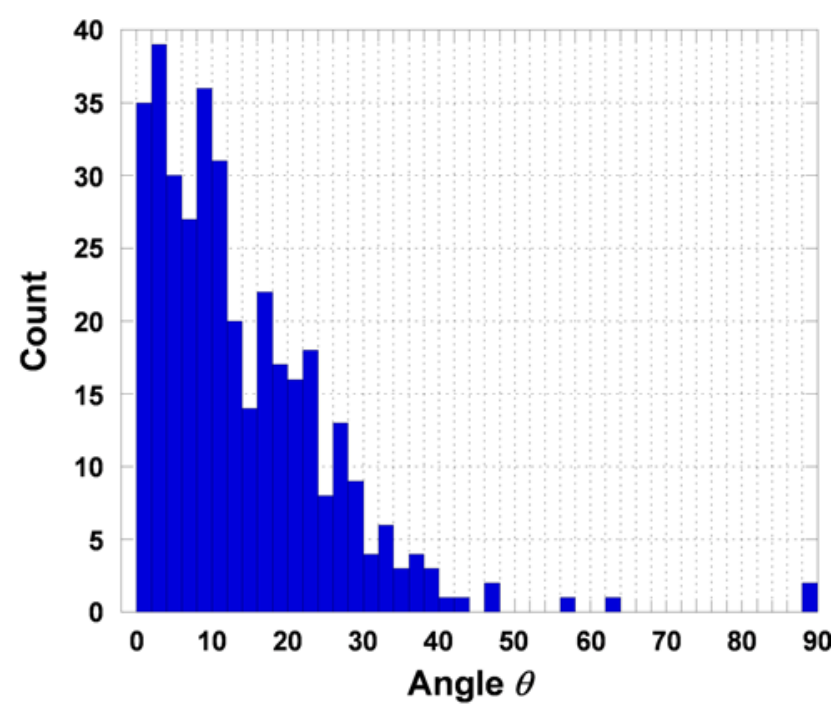

Figure S2. Histogram of the angular distribution (bin size: $2^{\circ}$ ) of $361 \mathrm{CdSe}$ NRs in the gap shown in Figure 3 of the main paper. The corresponding order parameter is $S=0.83$.

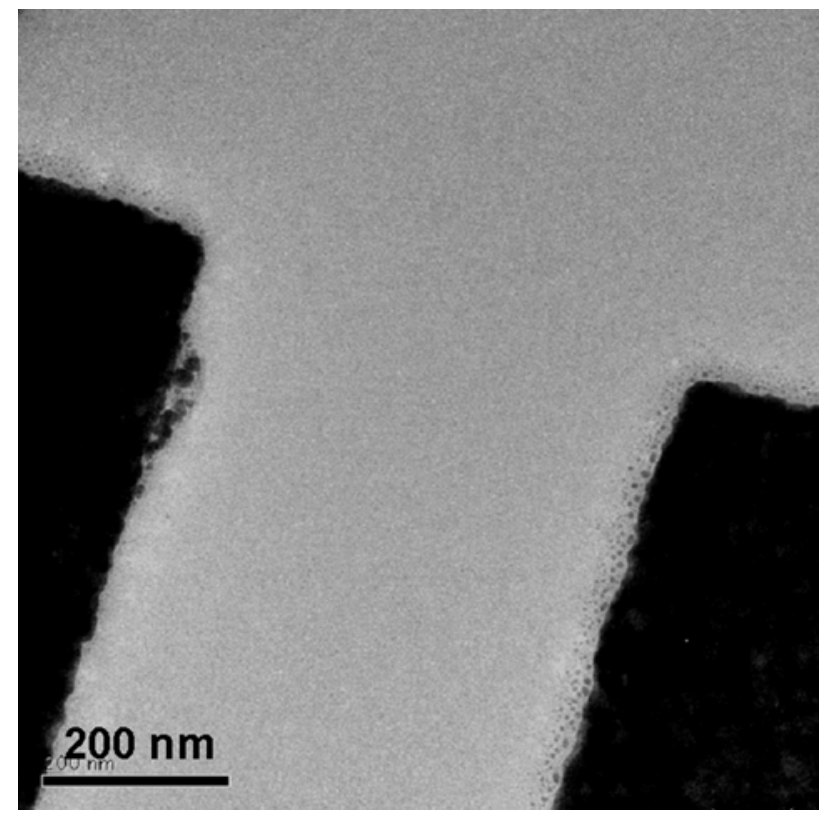

Figure S3. TEM image of a gap region between a "floating" electrode pair after deposition of $3.4 \times 38 \mathrm{~nm}$ CdSe NRs (concentration $\sim 5 \times 10^{13}$ rods $/ \mathrm{ml}$ ). At the time of deposition, the adjacent electrode pairs (not shown) were voltage-biased and showed dense accumulation ( $c f$. , Figure 5) 


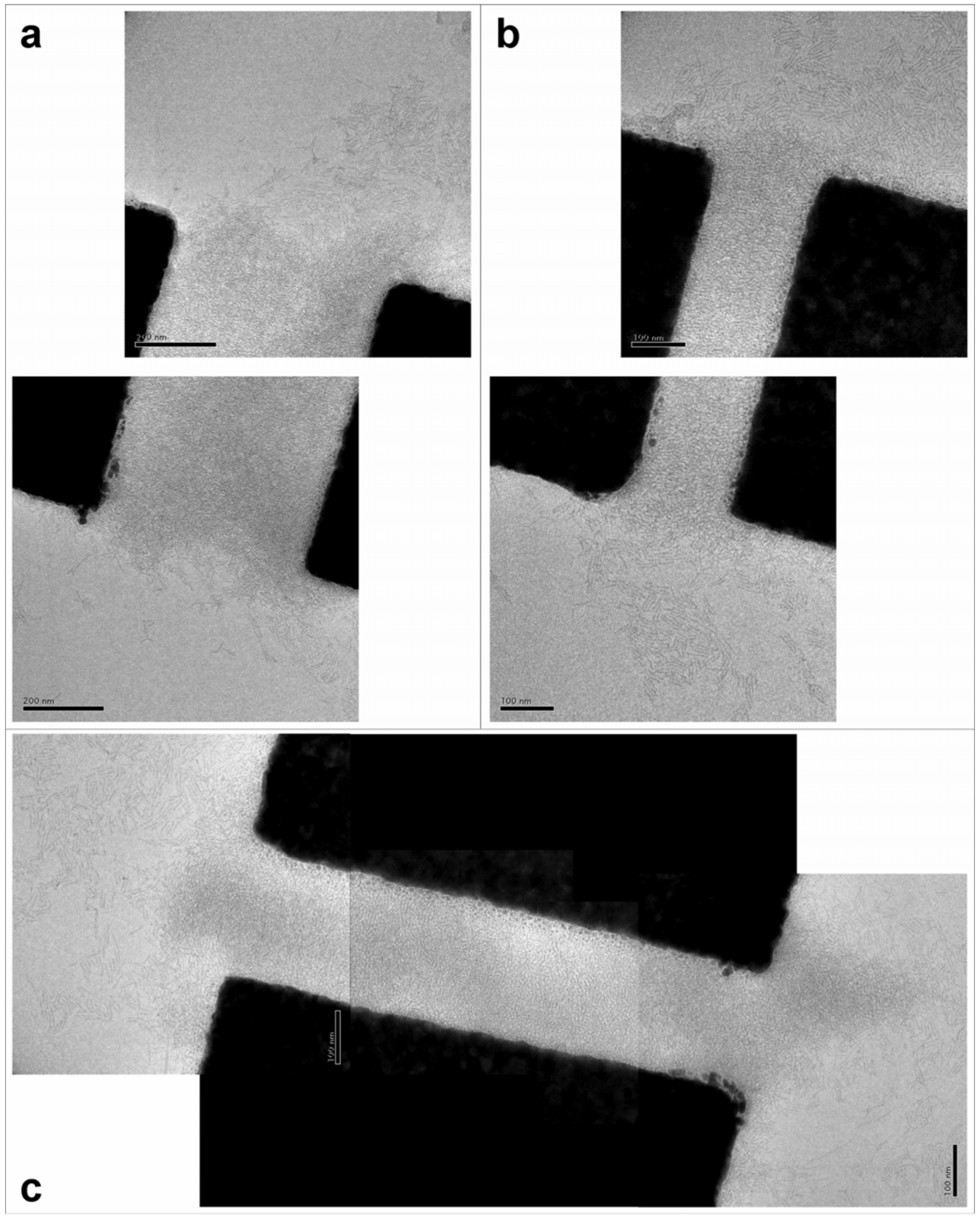

Figure S4. TEM images (created by pasting together several high magnification TEM images) of different electrode gaps after deposition of $3.4 \times 38 \mathrm{~nm} \mathrm{CdSe} \mathrm{NRs} \mathrm{(concentration} \sim 10^{14}$ rods $/ \mathrm{ml}$ ). For each gap, both electrodes were voltage-biased (opposite polarities) corresponding to an applied field of $E$ $\sim 2 \times 10^{7} \mathrm{~V} / \mathrm{m}$. 

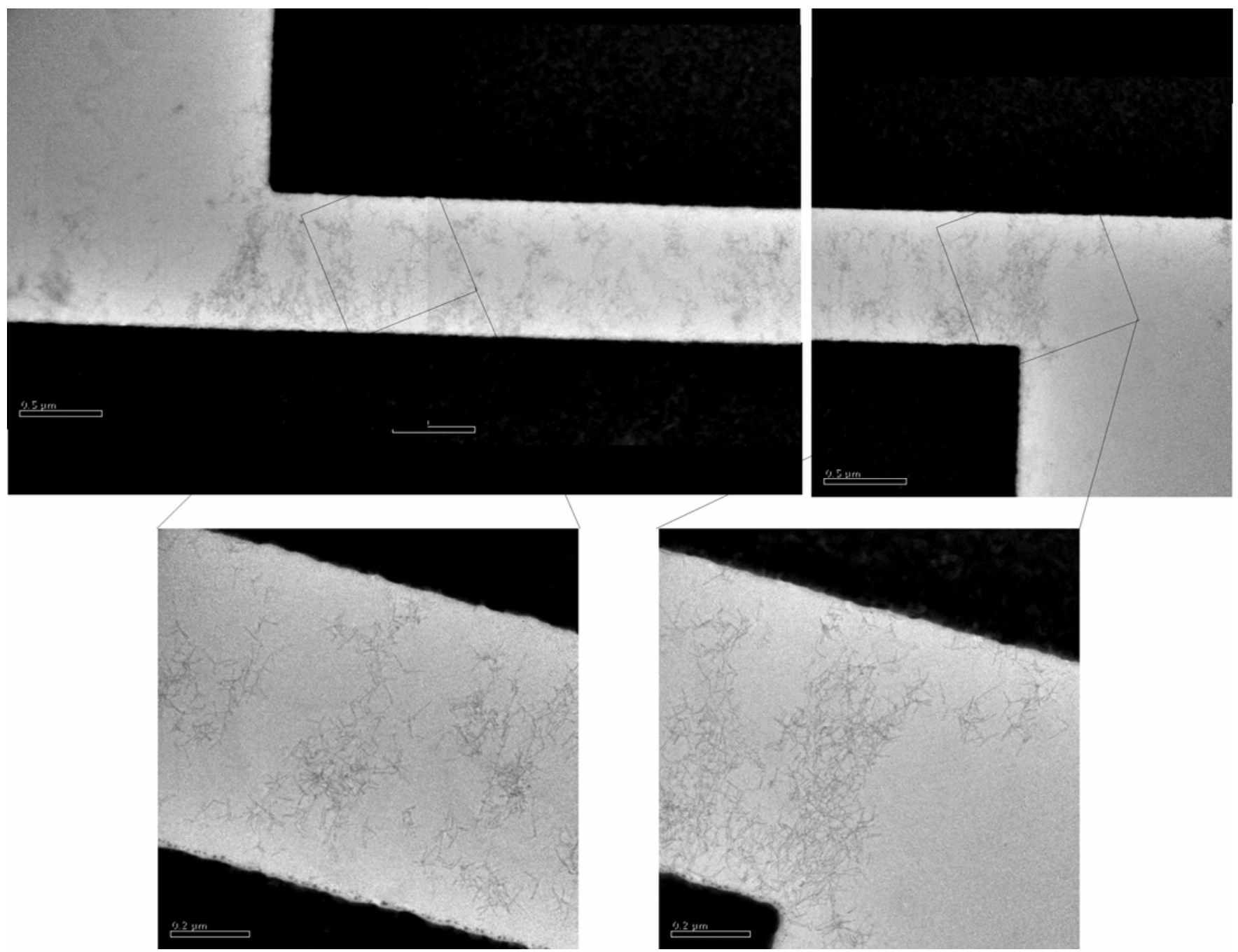

Figure S5. TEM images (created by pasting together several high magnification TEM images) of a device after deposition of branched CdTe NCs (concentration $\sim 10^{13} \mathrm{NCs} / \mathrm{ml}$ ). The applied field in the gap during deposition was $E=2 \times 10^{7} \mathrm{~V} / \mathrm{m}$. 